\title{
An optimised patient information sheet did not significantly increase recruitment or retention in a falls prevention study: an embedded randomised recruitment trial
}

\author{
Sarah Cockayne ${ }^{1}$, Caroline Fairhurst ${ }^{1}$, Joy Adamson ${ }^{1}$, Catherine Hewitt ${ }^{1}$, Robin Hull ${ }^{2}$, Kate Hicks ${ }^{1}$, \\ Anne-Maree Keenan ${ }^{3,4}$, Sarah E. Lamb ${ }^{5}$, Lorraine Green ${ }^{3,4}$, Caroline Mclntosh ${ }^{6}$, Hylton B. Menz ${ }^{7}$, \\ Anthony C. Redmond ${ }^{3,4}$, Sara Rodgers ${ }^{1}$, David J. Torgerson ${ }^{1}$, Wesley Vernon ${ }^{8}$, Judith Watson ${ }^{1}$, Peter Knapp ${ }^{9}$, \\ Jo Rick ${ }^{10^{*}}$ D, Peter Bower ${ }^{10}$, Sandra Eldridge ${ }^{11}$, Vichithranie W. Madurasinghe ${ }^{11}$ and Jonathan Graffy ${ }^{12}$
}

\begin{abstract}
Background: Randomised controlled trials are generally regarded as the 'gold standard' experimental design to determine the effectiveness of an intervention. Unfortunately, many trials either fail to recruit sufficient numbers of participants, or recruitment takes longer than anticipated. The current embedded trial evaluates the effectiveness of optimised patient information sheets on recruitment of participants in a falls prevention trial.
\end{abstract}

Methods: A three-arm, embedded randomised methodology trial was conducted within the National Institute for Health Research-funded REducing Falls with ORthoses and a Multifaceted podiatry intervention (REFORM) cohort randomised controlled trial. Routine National Health Service podiatry patients over the age of 65 were randomised to receive either the control patient information sheet (PIS) for the host trial or one of two optimised versions, a bespoke user-tested PIS or a template-developed PIS. The primary outcome was the proportion of patients in each group who went on to be randomised to the host trial.

Results: Six thousand and nine hundred patients were randomised 1:1:1 into the embedded trial. A total of 193 (2.8\%) went on to be randomised into the main REFORM trial (control $n=62$, template-developed $n=68$; bespoke user-tested $n=63$ ). Information sheet allocation did not improve recruitment to the trial (odds ratios for the three pairwise comparisons: template vs control 1.10 ( $95 \%$ Cl 0.77-1.56, $p=0.60)$; user-tested vs control 1.01 ( $95 \% \mathrm{Cl} 0.71-1.45, p=0.94)$; and user-tested vs template $0.92(95 \% \mathrm{Cl} 0.65-1.31, p=0.65)$ ).

Conclusions: This embedded methodology trial has demonstrated limited evidence as to the benefit of using optimised information materials on recruitment and retention rates in the REFORM study.

Trial registration: International Standard Randomised Controlled Trials Number registry, ISRCTN68240461. Registered on 01 July 2011.

Keywords: Recruitment, Patient information, Randomised controlled trial, Retention

\footnotetext{
* Correspondence: jo.rick@manchester.ac.uk

${ }^{10}$ Medical Research Council North West Hub for Trials Methodology Research,

National Institute of Health Research (NIHR) School for Primary Care

Research, Manchester Academic Health Science Centre, Centre for Primary

Care, University of Manchester, Oxford Road, Manchester M13 9PL, UK

Full list of author information is available at the end of the article
}

(c) The Author(s). 2017 Open Access This article is distributed under the terms of the Creative Commons Attribution 4.0 International License (http://creativecommons.org/licenses/by/4.0/), which permits unrestricted use, distribution, and reproduction in any medium, provided you give appropriate credit to the original author(s) and the source, provide a link to the Creative Commons license, and indicate if changes were made. The Creative Commons Public Domain Dedication waiver (http://creativecommons.org/publicdomain/zero/1.0/) applies to the data made available in this article, unless otherwise stated. 


\section{Background}

Randomised controlled trials (RCTs) are generally regarded as the 'gold standard' experimental design to determine the effectiveness of an intervention. Unfortunately, many trials either fail to recruit sufficient numbers of participants, or recruitment takes longer than anticipated [1]. Such difficulties not only have an impact on the power and external validity of the study's findings but may also increase the overall financial cost. Many trials implement methods to improve recruitment and/or retention such as the use of monetary incentives. There is, however, limited evidence as to the effectiveness of recruitment strategies in healthcare settings [2], and as a result, the UK Medical Research Council funded the Systematic Techniques for Assisting Recruitment to Trials (MRC START) research programme [3]. The aim of this programme was to "improve the evidence-base of recruitment to trials, enhance recruitment rates and make research more accessible to the public." One key objective of the project was to develop interventions to improve recruitment to trials and test them in embedded trials within ongoing 'host' trials. The initial focus was on testing optimised patient information sheets and multimedia resources.

Patient information sheets (PISs) are always given to potential trial participants along with verbal information as part of the informed consent process. A trial PIS has to be reviewed and approved by an ethics committee or an internal review body; nonetheless, there remain longstanding concerns regarding their length, complexity and the level of literacy skills required to understand the information, all of which may have a negative impact on recruitment [4]. Indeed, a recent sample of 20 PISs from recently completed or ongoing RCTs were found to be lengthy (mean word count 1853, standard deviation =960) and lacking information important to making an informed decision about trial participation [5]. However, Brierley et al. found that reducing the length of the PIS did not influence recruitment and actually yielded more ineligible patients [6]. Therefore, simply producing a shorter PIS is ineffective, and the content and interpretability of the information presented must be retained. One possible approach to improve the quality and appearance of PISs is to develop their content through a process of re-writing (for a lay audience), re-organisation, enhancing their appearance through input from a graphic designer and involving user testing $[7,8]$. The use of a professionally designed information pack was seen to have a small positive effect on initial response rates relative to a 'standard' pack $(2.7 \%$ difference in response rates, $95 \%$ confidence interval (CI) -0.06 to $5.5 \%, p=0.06)$ in an RCT embedded in an Avon Longitudinal Study of Parents and Children (ALSPAC) cohort [9]. However, undertaking commercial user testing and incorporating graphic design input into
PISs is costly, especially if the process needs to be completed for each trial; therefore, it has been suggested that a 'bank' of template PISs could be developed (which have been re-written and have undergone bespoke user testing and graphic design) in different populations. Researchers could select the most appropriate template on which to base their trial PIS according to their particular study population.

Embedding recruitment trials into ongoing trials is an efficient method to test recruitment strategies, and to help fill the knowledge gap with minimal resource use [10]. Table 1 presents a Consolidated Standards of Reporting Trials (CONSORT) checklist for reporting embedded recruitment trials. The REFORM study team were invited by the MRC START programme to embed a recruitment methodology trial within the REducing Falls with ORthoses and a Multifaceted podiatry intervention (REFORM) study. The REFORM study was selected, as the aims of the MRC START programme were compatible with both the need to maximise recruitment to the REFORM trial and the ethos of the York Trials Unit (YTU) to develop an understanding of effective recruitment interventions. The aim of the designed three-arm embedded trial was to determine if the number of participants recruited, randomised and retained to the REFORM trial could be improved by the use of both a bespoke usertested and a template-developed optimised PIS.

\section{Methods}

\section{Ethics approval}

This trial was embedded within the National Institute for Health Research (NIHR)-funded REFORM study [11], which aims to evaluate the clinical and cost effectiveness of a podiatry intervention for the prevention of falls in older people. Ethical approval for the REFORM study was given by NRES East of England - Cambridge East Research Ethics Committee and the University of York, Department of Health Sciences Research Governance Committee. Ethical approval for the PIS embedded methodology trial was given via a substantial amendment to the same committees. The embedded methodology trial is registered as a substudy to REFORM (ISRCTN68240461).

\section{Participant recruitment}

REFORM is a cohort RCT [12] in which patients were initially recruited to an observational cohort before potentially being randomised into an RCT. Electronic medical notes at participating National Health Service (NHS) podiatry clinics were searched to identify communitydwelling patients eligible for the REFORM observational cohort (i.e. those who were over the age of 65 and had attended a routine podiatry appointment within the past 6 months). Potentially eligible participants were mailed an invitation pack (letter of invitation, one of the three 
Table 1 Checklist of items for reporting embedded recruitment trials

\begin{tabular}{|c|c|c|}
\hline Section/topic and item no. & CONSORT 2010 (standard) checklist item & Extension for embedded recruitment trials \\
\hline \multicolumn{3}{|l|}{ Title and abstract } \\
\hline $1 a$ & Identification as a randomised trial in the title & $\begin{array}{l}\text { Identification as an embedded randomised recruitment } \\
\text { trial in the title }\end{array}$ \\
\hline $1 b$ & $\begin{array}{l}\text { Structured summary of trial design, methods, } \\
\text { results and conclusions (for specific guidance } \\
\text { see CONSORT for abstracts) }\end{array}$ & $\begin{array}{l}\text { Structured summary of embedded recruitment trial design, } \\
\text { methods, results and conclusions (for specific guidance } \\
\text { see CONSORT for abstracts) }\end{array}$ \\
\hline
\end{tabular}

Introduction

Background and objectives

$2 \mathrm{a}$

$2 b$

Methods

Trial design

3a

$3 b$

Participants

$4 a$

$4 b$

Interventions

5

Outcomes

$6 a$

$6 b$

Sample size

$7 a$

$7 b$

Randomisation

Sequence generation

$8 a$
The interventions for each group with sufficient details to allow replication, including how and when they were actually administered

Scientific background and explanation of rationale

Specific objectives or hypotheses

Description of trial design (such as parallel, factorial) including allocation ratio

Important changes to methods after trial commencement (such as eligibility criteria), with reasons

Eligibility criteria for participants

Settings and locations where the data were collected

Completely defined pre-specified primary and secondary outcome measures, including how and when they were assessed

Any changes to trial outcomes after the trial commenced, with reasons

How sample size was determined

When applicable, explanation of any interim analyses and stopping guidelines
Scientific background and explanation of rationale for embedded recruitment trial including a brief description of the host trial(s) as appropriate

Specific objectives or hypotheses for embedded recruitment trial

Description of embedded recruitment trial design (such as parallel, factorial, cluster) including allocation ratio

Important changes to methods of the embedded recruitment trial after commencement (such as eligibility criteria), with reasons

Eligibility criteria for participants for embedded recruitment trial, including any differences from those for the host trial(s)

Settings and locations where the embedded recruitment trial was carried out, including a brief description of the host trial(s) as appropriate

The interventions for each group (including control group) within the embedded recruitment trial with sufficient details to allow replication, including how, where and when they were actually administered

Completely defined pre-specified primary and secondary outcome measures for the embedded recruitment trial, including how and when they were assessed

Any changes to embedded recruitment trial outcomes after the embedded recruitment trial commenced, with reasons

How sample size for embedded recruitment trial was determined

When applicable, explanation of any interim analyses and stopping guidelines for embedded recruitment trial

Method used to generate the random allocation sequence for embedded recruitment trial 
Table 1 Checklist of items for reporting embedded recruitment trials (Continued)

$8 b$

Type of randomisation; details of any restriction (such as blocking and block size)

Allocation concealment mechanism

Mechanism used to implement the random allocation sequence (such as sequentially numbered containers), describing any steps taken to conceal the sequence until interventions were assigned

Implementation

10

Who generated the random allocation sequence, who enrolled participants and who assigned participants to interventions

Blinding

$11 a$

Statistical methods subgroup analyses and adjusted analyses

Results

Participant flow (a diagram is strongly recommended)

Statistical methods used to compare groups for primary and secondary outcomes

If done, who was blinded after assignment to interventions (for example, participants, care providers, those assessing outcomes) and how

If relevant, description of the similarity of interventions

Recruitment

$14 a$

Baseline data

15

Numbers analysed

16

Outcomes and estimation
A table showing baseline demographic and clinical characteristics for each group

For each group, number of participants (denominator) included in each analysis and whether the analysis was by original assigned groups

For each group, the numbers of participants who were randomly assigned, received For each group, losses and exclusions after randomisation, together with reasons

Dates defining the periods of recruitment and follow-up

Why the trial ended or was stopped

For each primary and secondary outcome results for each group, and the estimated effect size and its precision (such as 95\% confidence interval) intended treatment and were analysed for
Type of randomisation; details of any restriction (such as blocking and block size) in embedded recruitment tria

Mechanism used in the embedded recruitment trial to implement the random allocation sequence (such as sequentially numbered containers), describing any steps taken to conceal the sequence until interventions were assigned

Who generated the random allocation sequence(s), who enrolled participants and who assigned participants to embedded recruitment interventions

If done, who was blinded after assignment to embedded recruitment interventions (for example, participants, care providers, those assessing outcomes) and how

If relevant, description of the similarity of interventions in the embedded recruitment trial

Statistical methods used to compare groups for primary and secondary outcomes of the embedded recruitment trial

Methods for additional analyses, such as subgroup analyses and adjusted analyses for embedded recruitment trial

For each group in the embedded recruitment trial, the numbers of participants who were randomly assigned, received intended treatment and were analysed for the primary outcome

For each group, losses and exclusions after randomisation to embedded recruitment trial, together with reasons

Dates defining the periods of recruitment and follow-up for both embedded recruitment trial and host trial(s)

Why the embedded recruitment trial ended or was stopped

If possible a table showing baseline characteristics of each arm of the embedded recruitment trial

For each group in the embedded recruitment trial, number of participants (denominator) included in each analysis and whether the analysis was by original assigned groups

For each primary and secondary outcome, results for each group in the embedded recruitment trial, and the estimated effect size and its precision (such as 95\% confidence interval) 
Table 1 Checklist of items for reporting embedded recruitment trials (Continued)

\begin{tabular}{|c|c|c|}
\hline $17 b$ & $\begin{array}{l}\text { For binary outcomes, presentation of both } \\
\text { absolute and relative effect sizes is } \\
\text { recommended }\end{array}$ & $\begin{array}{l}\text { For binary outcomes in the embedded recruitment } \\
\text { trial, presentation of both absolute and relative } \\
\text { effect sizes is recommended }\end{array}$ \\
\hline \multicolumn{3}{|c|}{ Ancillary analyses } \\
\hline 18 & $\begin{array}{l}\text { Results of any other analyses performed, } \\
\text { including subgroup analyses and adjusted } \\
\text { analyses, distinguishing pre-specified } \\
\text { from exploratory }\end{array}$ & $\begin{array}{l}\text { Results of any other analyses performed for embedded } \\
\text { recruitment trial, including subgroup analyses and } \\
\text { adjusted analyses, distinguishing pre-specified } \\
\text { from exploratory }\end{array}$ \\
\hline \multicolumn{3}{|c|}{ Harms } \\
\hline 19 & $\begin{array}{l}\text { All important harms or unintended effects } \\
\text { in each group (for specific guidance } \\
\text { see CONSORT for harms) }\end{array}$ & $\begin{array}{l}\text { All important harms or unintended effects in each } \\
\text { group for both the embedded recruitment trial and } \\
\text { host trial(s) (for specific guidance see CONSORT } \\
\text { for harms) }\end{array}$ \\
\hline \multicolumn{3}{|c|}{ Discussion } \\
\hline \multicolumn{3}{|c|}{ Limitations } \\
\hline 20 & $\begin{array}{l}\text { Trial limitations, addressing sources of } \\
\text { potential bias, imprecision and, if relevant, } \\
\text { multiplicity of analyses }\end{array}$ & $\begin{array}{l}\text { Embedded recruitment trial limitations, addressing } \\
\text { sources of potential bias, imprecision and, if relevant, } \\
\text { multiplicity of analyses }\end{array}$ \\
\hline \multicolumn{3}{|c|}{ Generalisability } \\
\hline 21 & $\begin{array}{l}\text { Generalisability (external validity, applicability) } \\
\text { of the trial findings }\end{array}$ & $\begin{array}{l}\text { Generalisability (external validity, applicability) of } \\
\text { the embedded recruitment trial findings }\end{array}$ \\
\hline \multicolumn{3}{|c|}{ Interpretation } \\
\hline 22 & $\begin{array}{l}\text { Interpretation consistent with results, } \\
\text { balancing benefits and harms, and considering } \\
\text { other relevant evidence }\end{array}$ & $\begin{array}{l}\text { Interpretation consistent with results of the } \\
\text { embedded recruitment trial, balancing benefits } \\
\text { and harms, and considering other relevant evidence }\end{array}$ \\
\hline \multicolumn{3}{|c|}{ Other information } \\
\hline \multicolumn{3}{|c|}{ Registration } \\
\hline 23 & Registration number and name of trial registry & $\begin{array}{l}\text { Registration number and name of trial registry } \\
\text { (for all host trials and embedded recruitment trial } \\
\text { if available) }\end{array}$ \\
\hline \multicolumn{3}{|c|}{ Protocol } \\
\hline 24 & $\begin{array}{l}\text { Where the full trial protocol can be accessed, } \\
\text { if available }\end{array}$ & $\begin{array}{l}\text { Where the embedded recruitment trial protocol can } \\
\text { be accessed, if available }\end{array}$ \\
\hline \multicolumn{3}{|c|}{ Funding } \\
\hline 25 & $\begin{array}{l}\text { Sources of funding and other support } \\
\text { (such as supply of drugs), role of funders }\end{array}$ & $\begin{array}{l}\text { For embedded recruitment trial, sources of funding } \\
\text { and other support, role of funders and collaborators }\end{array}$ \\
\hline
\end{tabular}

PISs being evaluated in this embedded trial, consent form, screening questionnaire and prepaid envelope) asking whether they would like to participate in the REFORM study. The information and consent process covered all aspects of the cohort and trial, and so only one PIS was seen by participants whether they went on to be randomised into the REFORM trial or not. Participants who returned their completed consent and screening form to the YTU by post were assessed by researchers at the YTU for eligibility. Participants were ineligible if they reported neuropathy, dementia or another neurological condition such as Parkinson's or Alzheimer's disease; were unable to walk household distances without the help of a walking aid; or had a lower limb amputation or were unwilling to attend their local podiatry clinic. Eligible participants were sent a baseline questionnaire and monthly falls calendars. Participants who returned a baseline questionnaire and at least one monthly falls calendar (recording falls in the previous month) were included in the observational cohort. Those cohort participants who had either had one fall in the past 12 months or one fall in the past 24 months requiring hospital attention, or who reported a fear of falling on the baseline questionnaire (worried about falling at least some of the time in the past 4 weeks) were then eligible for randomisation to the REFORM trial. During the course of the trial, cohort participants who went on to have a fall could then become eligible for the REFORM trial. In order to minimise the delay between randomisation and the podiatrist seeing the participants in 
clinic for their REFORM appointment, participants were randomised when clinics had capacity and not at the point of becoming eligible for the trial.

At the point at which this embedded trial was nested within the host trial, the next podiatry clinics due to begin recruitment were Harrogate, Leeds, Scarborough, Selby and Sheffield. All patients due to be sent a REFORM invitation pack from these clinics were randomised to one of the three arms in the embedded methodology trial which determined which format of PIS they were sent.

\section{Randomisation and blinding}

This was an embedded, individually randomised, recruitment trial. A list of patients was generated and ordered by NHS number. Each patient was assigned a unique identification number. An independent data manager at the YTU, who was not involved in the recruitment of participants, generated the allocation sequence for the embedded methodology trial electronically. Randomisation was stratified by centre, using a single large block per site, which corresponded to its total sample size. Participants were allocated 1:1:1 to receive, in their invitation pack, either (1) the control PIS and control invitation letter; or (2) an optimised version of the PIS and invitation letter developed through bespoke user testing; or (3) an optimised template-developed PIS and the original invitation letter. Patients were then sent the allocated invitation pack by members of the research team based at the University of York. The researchers, patients and podiatrists were blind to the allocation. Patients who received the information sheet were unaware that they had been randomised to receive different information leaflets.

\section{Control group}

The 'control' PIS was the original PIS developed for the REFORM trial. It was written in accordance with the standard National Research Ethics Service template available at the time at which the study was set up. It underwent several revisions by the research team and was five pages of A4 paper in length. The invitation letter was one A4 page on NHS trust headed paper. The control PIS is shown in Additional file 1.

\section{Intervention group: 'bespoke user-tested' PIS}

The 'bespoke user-tested' PIS was developed in the following way: it was re-written for a lay audience (led by author PK), re-organised into eight subsections, had graphic design input and underwent user testing. The graphic design and user testing were both undertaken by commercial companies. Staff members who had considerable experience in writing patient material and a graphic designer revised the content and layout of the control PIS. A contents list was placed on the front page along with a list of key points about the study, with the main text divided into eight sections. Sentences and paragraphs were shortened and bullet points used for lists. The PIS then underwent three rounds of user testing, each involving 10 members of the public with limited podiatry- or trial-specific knowledge. During the usertesting process, the users (a group demographically similar to the REFORM trial target population but with no history of falls) were asked to find key pieces of information in the PIS relating to: the nature and purpose of the trial; the process and meaning of consent; and trial procedures and safety issues. Questions were arranged in such a way to ensure they did not match the order within the PIS. The final version of the PIS was printed on one sheet of double-sided A3 paper and folded to form a booklet. The control one-page invitation letter was revised and underwent user testing alongside the PIS, resulting in shortened text and the addition of bullet points to clarify what to do if the participant wished to take part. The user-tested PIS is shown in Additional file 2.

\section{Intervention group: 'template-developed' PIS}

The 'template-developed' PIS was written by three research fellows with more than 12 years' experience of recruiting patients to a range of randomised trials and who were currently working on the REFORM study. The content, layout and style were revised using a previously bespoke usertested PIS designed for an earlier trial conducted in a similar aged population [13]. In this process, the control PIS text was shortened and divided into seven sections, a contents list included and bullet points added to clarify what the participant should do if he/she wished to take part. A green colour scheme was used to match the trial logo. The PIS was reviewed and revised further by two other experienced researchers. The Public and Patient Involvement (PPI) group reviewed this PIS and gave feedback about its readability, layout, font size and content; however, no graphic design input or formal user testing was undertaken. The revised version was printed on one sheet of double-sided A3 paper and folded to form a booklet. Participants in the templatedeveloped PIS group were sent the same patient invitation letter as the control group. The template-developed PIS is shown in Additional file 3.

\section{Primary outcome}

The primary outcome was the proportion of patients in each group who went on to be randomised into the REFORM trial.

\section{Secondary outcomes}

Secondary outcomes were as follows: 
- Proportion of patients in each group who were recruited into the REFORM cohort

- Proportion of patients retained in the trial at 3 months post randomisation defined as returning at least the first 3 months' worth of falls calendars from the date of randomisation.

\section{Sample size}

As is usual with an embedded trial within a trial, no formal power calculation was undertaken for this embedded methodology trial, but the MRC START programme set out to include trials which planned to approach enough potential participants to allow 400 potential recruits to receive each recruitment intervention. Five centres were involved in the substudy; thus, the sample was constrained to only include participants eligible for the REFORM study who were due to be mailed a recruitment pack from these centres.

\section{Statistical analysis}

The proportion of participants who: returned a consent form; were recruited into the cohort; were randomised into the main trial; and were retained in the trial is presented for the three groups. Odds ratios (ORs) and 95\% confidence intervals (CIs) for each of the three pairwise comparisons were obtained from mixed logistic regression models adjusting for PIS allocation as a fixed effect and trial centre as a random effect. Analysis was conducted in Stata v13, using two-sided tests at the 5\% significance level and based on the principles of intention to treat.

To account for the participants in the embedded trial of the newsletter who overlapped with participants in this trial, sensitivity analyses were conducted including a covariate for receiving a newsletter in all logistic regressions.

\section{Results}

A total of 6900 recruitment packs were sent to potential REFORM study participants across five centres between March 2013 and May 2014: Harrogate $(n=500,7.3 \%)$, Scarborough $(n=1000,14.5 \%)$, Selby $(n=1000,14.5 \%)$, Sheffield $(n=1600,23.2 \%)$ and Leeds $(n=2800,40.6 \%)$ (Fig. 1). Potential participants were sent a recruitment pack containing either the control version of the PIS $(n=2298,33.3 \%)$, the template-developed PIS $(n=$ $2301,33.4 \%)$ or the user-tested PIS ( $n=2301,33.4 \%)$.

\section{Responses to invitation}

A consent form and screening questionnaire were returned by 755 or $10.9 \%$ of individuals (control $n=254$ (11.1\%); template-developed $n=246$ (10.7\%); user-tested $n=255(11.1 \%))$. PIS allocation did not significantly improve the response (chi-square 0.22, degrees of freedom $=2, p=0.89$ ). No one group was more likely to respond

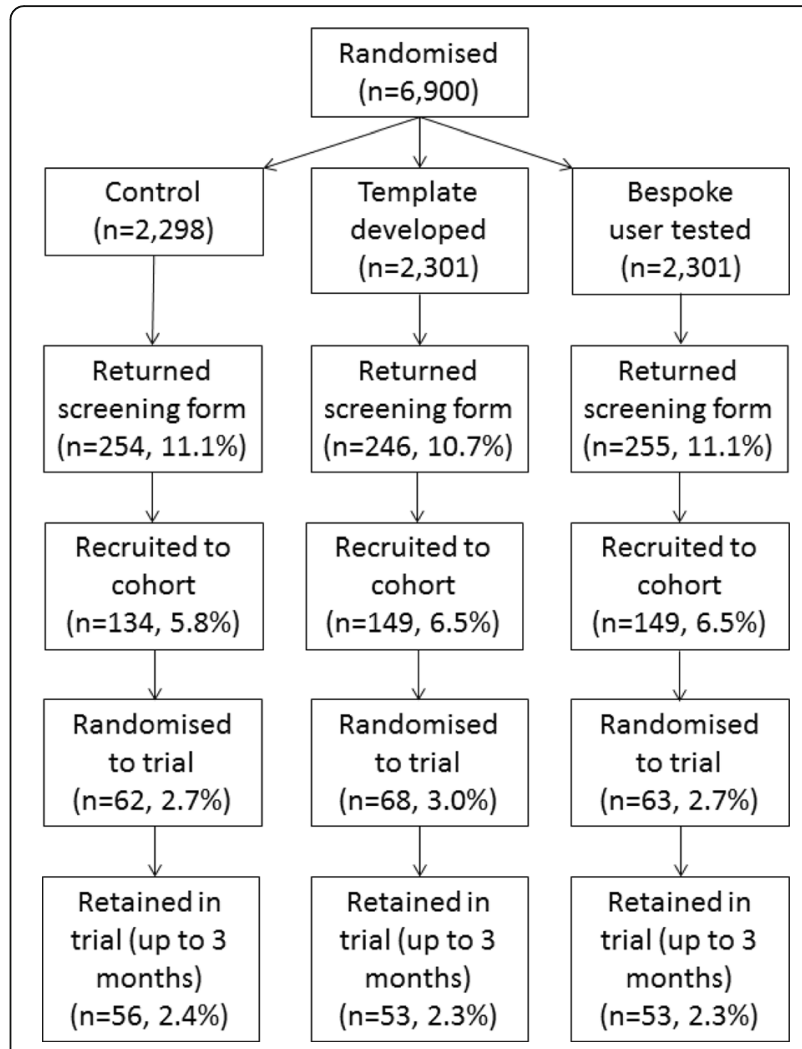

Fig. 1 Flow diagram to depict the flow of participants in the PIS embedded methodology trial

(template-developed vs control OR 0.96, 95\% CI 0.80 1.16, $p=0.69$; bespoke user-tested vs control OR 1.00 , 95\% CI $0.83-1.21, p=0.98$; and bespoke user-tested vs template-developed OR 1.04, 95\% CI 0.86-1.25, $p=0.67$ ).

\section{Randomised to REFORM trial}

Eligible, consenting participants were then sent a baseline questionnaire (control $n=145$ (6.3\%); templatedeveloped $n=158$ (6.9\%); bespoke user-tested $n=160$ $(7.0 \%))$. A baseline form and at least one falls calendar were returned by $134(5.8 \%)$ individuals in the control group, $149(6.5 \%)$ in the template-developed group and 149 (6.5\%) in the bespoke user-tested group. These participants were recruited to the cohort of potentially eligible participants.

Of these cohort patients, 161 were immediately eligible for randomisation into REFORM, and of these, 122 could be randomised as there was sufficient capacity in the clinics to see them. A further 71 patients in the cohort became eligible for the trial as they subsequently reported a fall and were able to be seen in clinic. Therefore, a total of 193 of the 6900 potential participants (2.8\%) went on to be randomised into the main REFORM trial (control $n=62(2.7 \%, 95 \%$ CI $2.0-3.4 \%$ ); template-developed $n=68$ (3.0\%, 95\% CI $2.3-3.6 \%)$; 
bespoke user-tested $n=63(2.7 \%, 95 \%$ CI 2.1-3.4\%)). The difference in percentages and their 95\% CIs are as follows: for template-developed vs controls there was a 0.3 percentage point difference $(95 \% \mathrm{CI}-0.7$ to $1.2, p=$ 0.60 ); bespoke user-tested vs control 0.0 ( $95 \% \mathrm{CI}-0.9$ to 1.0, $p=0.93)$; and bespoke user-tested vs templatedeveloped -0.2 ( $95 \% \mathrm{CI}-1.2$ to $0.7, p=0.66$ ).

Descriptive data for these participants are presented in Table 2. PIS allocation did not improve recruitment to the trial (chi-square 0.33, degrees of freedom $=2, p=0.85$ ). The odds ratios for the three pairwise comparisons were: template-developed vs control $1.10(95 \%$ CI $0.77-1.56, p=0.60)$; bespoke user-tested vs control 1.01 (95\% CI $0.71-1.45, p=0.94)$; and bespoke usertested vs template-developed 0.92 (95\% CI $0.65-1.31$, $p=0.65)$.

\section{Retention rates}

In total, 162 participants were retained in the trial (control $n=56$ (2.4\%); template-developed $n=53$ (2.3\%); user-tested $n=53(2.3 \%))$.

\section{Sensitivity analyses}

Three hundred and thirty four participants also received a newsletter in their recruitment pack (control $n=120$
(5.2\%); template-developed $n=112$ (4.9\%); bespoke usertested $n=102(4.4 \%))$. In the sensitivity analyses, only a negligible difference in the estimates, their associated 95\% CIs and $p$ values was observed.

\section{Discussion}

We have evaluated the effectiveness of two optimised patient information sheets (PISs) relative to the standard control version developed using the NRES template PIS to increase recruitment to the REFORM trial. Previous studies investigating the method of delivering paperbased information to patients have found that reducing the length of the PIS or supplementing the PIS with a booklet on clinical trials has little or no impact on trial recruitment, but that the use of a professionally designed information pack may be effective $[6,9,14]$. Alternative strategies could be evaluated in future embedded trials. Bower et al. [10] have identified three areas which merit further investigation. These include training site staff, communication with patients and incentives. The key finding of this study is that the use of enhanced PISs did not significantly increase recruitment or retention. The overall recruitment rate to the REFORM trial (2.8\%) and the REFORM cohort (6.3\%) was low, so it may be that there was insufficient power to detect a difference. The

Table 2 Characteristics of participants randomised into the main trial by PIS allocation

\begin{tabular}{|c|c|c|c|c|}
\hline Characteristic & Control $(n=62)$ & Template-developed $(n=68)$ & Bespoke user-tested $(n=63)$ & Total $(n=193)$ \\
\hline \multicolumn{5}{|l|}{ Gender, $n(\%)$} \\
\hline Male & $24(38.7)$ & $32(47.8)$ & $27(43.6)$ & $83(43.5)$ \\
\hline \multicolumn{5}{|l|}{ Age } \\
\hline Mean (SD) & $78.3(5.8)$ & $78.6(6.6)$ & $77.5(7.8)$ & $78.1(6.8)$ \\
\hline \multicolumn{5}{|c|}{ Fallen in previous 6 months? $n(\%)$} \\
\hline Yes & $23(37.1)$ & $29(43.3)$ & $27(42.9)$ & $79(41.2)$ \\
\hline No & $39(62.9)$ & $36(53.7)$ & $36(57.1)$ & $111(57.8)$ \\
\hline Don't know & $0(0.0)$ & $2(3.0)$ & $0(0.0)$ & $2(1.0)$ \\
\hline \multicolumn{5}{|c|}{ If fallen in previous 6 months, how many times? } \\
\hline Median (min,max) & $1(1,5)$ & $1(1,20)$ & $1(1,6)$ & $1(1,20)$ \\
\hline \multicolumn{5}{|c|}{ Worried about having a fall during the previous 4 weeks } \\
\hline All of the time & $4(6.5)$ & $3(4.5)$ & $4(6.4)$ & $11(5.7)$ \\
\hline Most of the time & $1(1.6)$ & $4(6.0)$ & $2(3.2)$ & $7(3.7)$ \\
\hline A good bit of the time & $6(9.7)$ & $3(4.5)$ & $4(6.4)$ & $13(6.8)$ \\
\hline Some of the time & $19(30.7)$ & $13(19.4)$ & $9(14.3)$ & $41(21.4)$ \\
\hline A little of the time & $23(37.1)$ & $27(40.3)$ & $29(46.0)$ & $79(41.2)$ \\
\hline None of the time & $9(14.5)$ & $17(25.4)$ & $15(23.8)$ & $41(21.4)$ \\
\hline \multicolumn{5}{|c|}{ Short Falls Efficacy Scale - International (FES-I) } \\
\hline Mean (SD) & $13.1(5.0)$ & $11.7(4.3)$ & $12.1(4.5)$ & $12.3(4.7)$ \\
\hline \multicolumn{5}{|c|}{ Frenchay Activities Index (FAI) ${ }^{a}$} \\
\hline Mean (SD) & $47.2(6.7)$ & $46.5(7.2)$ & $46.4(7.1)$ & $46.6(7.0)$ \\
\hline
\end{tabular}

${ }^{\mathrm{a}} \mathrm{FES}-\mathrm{I}$ scored from 7-28, higher score indicates greater concern about the possibility of falling; FAl scored from 15-60, higher score indicates greater activity 
MRC START group will undertake a meta-analysis of six embedded trials evaluating enhanced information sheets which will have more power to provide evidence of effectiveness. Small differences in recruitment rates could make a large difference to trials similar to REFORM, which required a sample size of nearly 900 participants. For example, a 3\% uptake would require 30,000 invitations, but if uptake to the study increased to $4 \%$, then 7500 fewer recruitment packs would be required, saving up to $£ 35,000$ in packing and postage costs alone. This would be a significant saving, even after incorporating the cost of professional graphic design and bespoke user testing. In this study, there were no statistically significant differences between the bespoke user-tested and template-developed information sheets, so it may be beneficial to develop a bank of template information sheets rather than each trial having to take account of the additional time and expense of professional user testing. Alternatively, since many trials include PPI, a more structured approach to reviewing and developing the information sheet could be evaluated and then, if effective, adopted within PPI groups.

One reason why there may have been little evidence of a difference is that the 'standard' PIS has been developed by experienced researchers, and consequently it may be difficult to achieve a significantly improved information sheet. It could also be that the optimised PISs increased the interpretably and clarity of the provided information, which deterred some patients from responding. Unlike the other host trials in the START programme, REFORM used a cohort randomised controlled trial design. Participants were recruited first to the observational cohort, and then eligible participants were randomised to the REFORM trial. Therefore, optimised PISs were more likely to have an effect on recruitment to the observational cohort than randomisation into the REFORM trial. As there was a lack of effect on recruitment to the observational cohort, it was unlikely that there would be an impact on recruitment or retention in the trial.

There are some potential limitations to this study. First, the results of this study are applicable only to those participants over the age of 65 years attending routine podiatry clinics. Further studies should substantiate the study results in other populations. Second, due to the nature of the intervention, it was not possible to blind administrative staff who mailed out the recruitment packs, but it is unlikely that allocation subversion could have taken place. Whilst participants were aware of which PIS they received, they were unaware that an embedded trial was being conducted and that recruitment to the REFORM study was being monitored. Finally, it was not possible to evaluate whether the enhanced information sheets affected the speed of recruitment, as podiatry clinics were limited to the number of participants they could see at any one time.

\section{Conclusions}

Optimised participant information sheets are one of a number of recruitment interventions amenable to testing through embedded trial methodology. Whilst the results of this study suggest limited benefits to enhancing patient information sheets, the findings add to the body of evidence around the effectiveness of recruitment strategies and may potentially help save time and money in future trials. The results of this study and the other embedded studies for the MRC START programme are to be incorporated into a meta-analysis which will provide more robust evidence as to the effectiveness of these types of recruitment strategies.

\section{Guidelines for reporting embedded recruitment trials}

The guidelines are obtained from the following source: Vichithranie W. Madurasinghe and Sandra Eldridge on behalf of the MRC START group and Gordon Forbes on behalf of the START Expert Consensus group. Trials. 2016;17:27. Published online 2016 Jan 14. doi: 10.1186/ s13063-015-1126-y.

\section{Additional files}

\author{
Additional file 1: Original, control patient information sheet. (DOC $103 \mathrm{~kb}$ ) \\ Additional file 2: Bespoke user-tested patient information sheet. \\ (PDF $328 \mathrm{~kb}$ )
}

Additional file 3: Template-developed patient information sheet. (PDF $162 \mathrm{~kb}$ )

\section{Abbreviations}

ALSPAC: Avon Longitudinal Study of Parents and Children; Cl: Confidence interval; ISRCTN: International Standard Randomised Controlled Trial Number; MRC START: Medical Research Council Systematic Techniques for Assisting Recruitment to Trials; NHS: National Health Service; NRES: National Research Ethics Service; OR: Odds ratio; PIS: Patient information sheet; PPI: Public and Patient Involvement; RCT: Randomised controlled trial; REFORM: REducing Falls with ORthoses and a Multifaceted podiatry intervention; YTU: York Trials Unit

\section{Acknowledgements}

The authors are grateful to all the REFORM participants who took part in the study. We thank the MRC START group for funding the user testing and the graphic design of the patient information sheet. We thank Luto

(www.luto.co.uk) and Making Sense Design (www.makingsense.co.uk) for their contributions to user testing and graphic design, respectively, of the trial patient information sheets. We would like to thank members of our PPI group for reviewing and giving feedback on the patient information sheet.

The University of York is the study sponsor and has legal responsibility for the initiation and management of the study: sponsor representative Mrs Sue Final, Research Innovation Office, York Science Park, Heslington, York UK YO10 5DG.

\section{Funding}

The REFORM study is funded by the National Institute for Health Research (NIHR) Health Technology Assessment (HTA) Programme (Programme grant number 09/77/01). The views and opinions expressed herein are those of the authors and do not necessarily reflect those of the Department of Health. The MRC START programme is funded by the MRC Methodology Research Programme (MRP). Materials relating to the MRC START programme can be found in the protocol and on the University of Manchester website [3] (http://www.population-health.manchester.ac.uk/mrcstart/). 


\section{Availability of data and materials}

Requests to access the dataset can be made to the corresponding author, and will be considered on a case-by-case basis by the Trial Management Group. All data requests will be managed according to York Trials Unit, University of York processes and procedures.

\section{Authors' contributions}

SC wrote the patient information sheet and the embedded trial protocol, prepared the template version of the optimised PIS and drafted the manuscript. CF conducted the statistical analysis and drafted the manuscript. JA participated in the design of the embedded study and reviewed the protocol. CH participated in the design of the embedded study and reviewed the protocol and statistical analysis plan. $\mathrm{RH}$ participated in the design of the embedded study. KH participated in the design and coordination of the embedded study. AMK participated in the design of the embedded study. SL participated in the design of the embedded study. LG participated in the design and coordination of the embedded study. CM participated in the design of the embedded study. HBM participated in the design of the embedded study and reviewed the protocol. ACR participated in the design of the embedded study. SR prepared the template version optimised PIS, participated in the design and coordination of the study and reviewed the protocol. DT is a Co-investigator of the MRC START programme and Chief Investigator of the REFORM study, conceived of the embedded study, participated in its design and coordination and reviewed the protocol. WW participated in the design of the embedded study. JW prepared the template version optimised PIS. PK is a Co-investigator of the MRC START programme and led the user testing and graphic design for one of the optimised PISs. JR was the trial manager of the MRC START programme and participated in the preparation and of the protocol and design of the embedded study. PB is the Principal Investigator of the MRC START programme. $\mathrm{SE}$ is a Co-investigator of the MRC START programme and prepared the standard analytic plan and reporting guidelines as part of the MRC START programme which was used to inform the statistical analysis and reporting of the embedded study. WW prepared the standard analytic plan and reporting guidelines as part of the MRC START programme which was used to inform the statistical analysis and reporting of the embedded study. JG is a Co-investigator of the MRC START programme. All authors read and approved the final manuscript.

\section{Authors' information}

\section{Not applicable.}

\section{Competing interests}

SC, JA, CH, RH, AMK, SL, CM, HBM, ACR, DT, WW and JW were applicants on the NIHR HTA funding application. DT and SC wrote the original REFORM trial protocol. The NIHR grant application included some salary costs for SC, $C F, J A, C H, R H, K H, A M K, S L, L G, C M, H B M, A C R, S R$, DT, WW and JW. The authors declare that they have no competing interests.

\section{Consent for publication}

\section{Not applicable.}

\section{Ethics approval and consent to participate}

The embedded study was approved by the NRES East of England - Cambridge East Research Ethics Committee (REC Ref No.11/EE/0379, February 2013) and the University of York, Department of Health Sciences Research Governance Committee. All participants provided their informed, written consent to participate.

\section{Author details}

${ }^{1}$ Department of Health Sciences, York Trials Unit, University of York, York YO10 5DD, UK. ${ }^{2}$ Podiatry Services, Harrogate and District NHS Foundation Trust, Harrogate District Hospital, Lancaster Park Road, Harrogate, UK. ${ }^{3}$ NIHR Leeds Musculoskeletal Biomedical Research Unit, Chapel Allerton Hospital, Leeds, UK. ${ }^{4}$ Leeds Institute of Rheumatology and Musculoskeletal Medicine, University of Leeds, Leeds, UK. ${ }^{5}$ Nuffield Department of Orthopaedics, Rheumatology and Musculoskeletal Sciences, Kadoorie Critical Care Research Centre, John Radcliffe Hospital, University of Oxford, Oxford, UK. 'School of Health Sciences, Aras Moyola, National University of Ireland, Galway, Ireland. 7Lower Extremity and Gait Studies Program, Faculty of Health Sciences, La Trobe University, Bundoora 3086, Victoria, Australia. ${ }^{8}$ The School of Human \&
Health Sciences, Division of Podiatry, University of Huddersfield, Huddersfield, UK. ${ }^{9}$ Department of Health Sciences and the Hull York Medical School, University of York, York YO10 5DD, UK. ${ }^{10}$ Medical Research Council North West Hub for Trials Methodology Research, National Institute of Health Research (NIHR) School for Primary Care Research, Manchester Academic Health Science Centre, Centre for Primary Care, University of Manchester, Oxford Road, Manchester M13 9PL, UK. ${ }^{11}$ Pragmatic Clinical Trials Unit (PCTU), Centre for Primary Care and Public Health, Blizard Institute, Yvonne Carter Building, 58 Turner Street, London E1 2AB, UK. ${ }^{12}$ Department of Public Health and Primary Care, University of Cambridge, Institute of Public Health, Forvie Site, Robinson Way, Cambridge CB2 OSR, UK.

Received: 8 February 2016 Accepted: 11 January 2017 Published online: 28 March 2017

\section{References}

1. McDonald A, Knight R, Campbell M, et al. What influences recruitment to randomised controlled trials? A review of trials funded by two UK funding agencies. Trials. 2006;7:9. doi:10.1186/1745-6215-7-9.

2. Treweek $\mathrm{S}$, Lockhart $P$, Pitkethly $\mathrm{M}$, et al. Methods to improve recruitment to randomised controlled trials: Cochrane systematic review and meta-analysis. BMJ Open. 2013;3(2). doi:10.1136/bmjopen-2012-002360.

3. Rick J, Graffy J, Knapp P, et al. Systematic Techniques for Assisting Recruitment to Trials (START): study protocol for embedded, randomized controlled trials. Trials. 2014;15(1):407.

4. Terblanche $M$, Burgess $L$. Examining the readability of patient-informed consent forms. J Clin Trials. 2010;2:157-62.

5. Gillies K, Huang W, Skea Z, et al. Patient information leaflets (PILs) for UK randomised controlled trials: a feasibility study exploring whether they contain information to support decision making about trial participation. Trials. 2014;15(1):62.

6. Brierley $G$, Richardson $R$, Torgerson DJ. Using short information leaflets as recruitment tools did not improve recruitment: a randomized controlled trial. J Clin Epidemiol. 2012;65(2):147-54. http//dx.doi.org/10.1016/j.jclinepi.2011.06.005.

7. Knapp P, Raynor DK, Silcock J, et al. Can user testing of a clinical trial patient information sheet make it fit-for-purpose? - a randomized controlled trial. BMC Med. 2011:9(1):89.

8. Raynor DK, Knapp P, Silcock J, et al. "User-testing" as a method for testing the fitness-for-purpose of written medicine information. Patient Educ Couns. 2011;83(3):404-10.

9. Boyd $A$, Tilling $K$, Cornish $R$, et al. Professionally designed information materials and telephone reminders improved consent response rates: evidence from an RCT nested within a cohort study. J Clin Epidemiol. 2015; 68(8):877-87. http://dx.doi.org/10.1016/j.jclinepi.2015.03.014.

10. Bower P, Brueton V, Gamble C, et al. Interventions to improve recruitment and retention in clinical trials: a survey and workshop to assess current practice and future priorities. Trials. 2014;15(1):399.

11. Cockayne S, Adamson J, Corbacho Martin B, et al. The REFORM study protocol: a cohort randomised controlled trial of a multifaceted podiatry intervention for the prevention of falls in older people. BMJ Open. 2014; 4(12). doi:10.1136/bmjopen-2014-006977.

12. Relton $C$, Torgerson $D, O^{\prime} C a t h a i n ~ A$, et al. Rethinking pragmatic randomised controlled trials: introducing the "cohort multiple randomised controlled trial" design. BMJ. 2010;340:c1066.

13. Mitchell N, Hewitt C, Adamson J, et al. A randomised evaluation of CollAborative care and active surveillance for Screen-Positive EldeRs with sub-threshold depression (CASPER): study protocol for a randomized controlled trial. Trials. 2011;12(225):6215-12.

14. Ives NJ, Troop M, Waters A, et al. Does an HIV clinical trial information booklet improve patient knowledge and understanding of HIV clinical trials? HIV Med. 2001;2(4):241-9. 